\section{Thermo-responsive fibrinogen nanogels: a viable thermo-responsive drug delivery agent for breast cancer therapy?}

\author{
"In the last few years, many researchers have reported the use of this \\ nanoformulated natural protein as a drug cargo for cancer."
}

Keywords: breast cancer $\bullet$ cancer nanomedicine $\bullet$ drug delivery $\bullet$ fibrinogen

- thermo-responsive nanogel

Breast cancer is reported to be the most frequent cancer type in women worldwide, with approximately 1.7 million newly diagnosed cases reported in 2012. During their lifetime, approximately $12 \%$ of women in the USA will develop invasive breast cancer, the second leading cause of cancer death of women in the USA [1]. In India, 1000,000 new cases of breast cancer are being reported every year [2]. Both local and systemic therapies are available for breast cancer now. Current treatment methods for breast cancer include invasive surgical procedures, radiotherapy, hormone therapy and chemotherapy. These therapies are less effective and recurrence is still a major problem in breast cancer patients. These therapies impart severe side effects and significant toxicity to normal cells [3]. A minimally invasive local delivery system capable of delivering one or a combination of drugs may overcome risks associated with surgery and also reduce the toxicity of anticancer drugs to normal cells/tissues. Nanogel holds promise as one of the best drug-delivery systems owing to its water solubility, biocompatibility, excellent encapsulation stability and ease to synthesize. Nanogels also respond well to biological stimuli. Various possibilities of using nanogels exist in cancer therapeutics as unlike other commonly available cancer chemotherapeutic drugs used in the clinic, which target cancer cells from outside, nanogels can be effectively used to functionalize with ligands and that in turn helps in cancer cell targeting [4-6].
For carrying therapeutic moieties for cancer, the usage of macromolecules such as fibrin and fibrinogen are gaining interest nowadays. Fibrin glue has been exploited in carrying methotraxate, which showed considerable potential in shrinking tumors in glioblastoma in vivo. Fibrinogen-bound methotrexate therapy in vivo was described for Gardner lymphosarcoma in mice. Fibrinogen-methotrexate conjugate showed significant in vivo anti-tumor activity compared with that of free methotrexate and thus suggested the therapeutic utility of these potential drug conjugates [7]. Jakate et al. reported docetaxelloaded olive oil droplet coated with fibrinogen useful for taxane-sensitive fibrin-rich tumors by facilitating the retention of these droplets in the tumor microenvironment. They reported improvement in the median survival time of B16F10 melanoma bearing mice compared with the free taxotere treatment [8].

In the last few years, many researchers have reported the use of this nanoformulated natural protein as a drug cargo for cancer. Sanoj et al. reported the fabrication of biocompatible fibrinogen nanoparticle (FNP) using simple co-acervation method. The $150 \mathrm{~nm}$-sized fibrinogen nanoparticles was taken up efficiently by the cancer cells and nontoxic to an array of cancer and normal cell lines. FNPs were also found to be hemocompatible and biocompatible in vitro [9].

These fibrinogen nanoparticles were used for delivering anticancer agents like curcumin (CRC), a potent phytochemi-
Maya Sreerenganathan', Ullas Mony $y^{\ddagger}, 1$ \& Jayakumar Rangasamy ${ }^{\ddagger, *, 1}$

${ }^{1}$ Amrita Centre for Nanosciences \& Molecular Medicine, Amrita Institute of Medical Sciences \& Research Centre, Kochi, India

*Author for correspondence: rjayakumar@aims.amrita.edu 
cal and 5-flurouracil (5-FU), a pyramidine analog. CRC-FNPs and 5-FU-FNPs exhibited controlled and sustained release of these drugs in vitro and induced toxicity toward breast cancer cell lines (MCF-7). The tumor-accumulating property of fibrinogen is utilized and hence advantageous for delivering these anticancer therapeutic agents [10,11]. There are reports regarding the peptides binding to the irradiated tumor microvasculature and hence conjugation of these peptides to various carrier systems aid in tumor targeted drug delivery. The affinity of fibrinogen to integrin receptors has been studied in B16F0 tumors. Fibrinogen nanoparticles and liposomes were found to selectively bind within irradiated tumor blood vessels. The study reported the binding of fibrinogen-conjugated nanoparticles to radiation-activated receptors, reduced tumor blood flow and showed significant delayed and regressed tumor growth [12,13]. The above-mentioned micronized olive oil droplet with docetaxel and fibrinogen coating was also found to be effective against TA3/St mammary tumor grown in ascites [8].

\section{"A multifunctional fibrinogen nanoparticle was reported by Sanoj et al. for simultaneous therapy and imaging of breast cancer cells in vitro."}

Now researchers are thinking of stimuli-sensitive nanosystems for the efficient release of drugs to the target. Nanogels containing water within their structures hold promise in biomedical applications due to their responsiveness to temperature $[13,14]$. Fibrinogen was again exploited in thermo-responsive delivery of breast cancer drugs like megestrol acetate (Meg) in targeted fashion. Thermo-responsive polymer-like $\operatorname{poly}(N$-isopropylacrylamide) (PNIPAAm) with a lower critical solution temperature (LCST) in the range of $30-32^{\circ} \mathrm{C}$ was grafted with fibrinogen and nanoformulated into nanogels with a combination of 5-FU/Meg drugs loaded within. Here the affinity of fibrinogen to interact with $\alpha 5 \beta 1$ Integrin receptors overexpressed on various cancer cells including breast cancer cells has been reported. Thus 5-FU/Meg-loaded-fib-graft-PNIPAAm NGs preferentially target and deliver the therapeutic

\section{References}

1 American Cancer Society. Facts and Figures (2014). www.cancer.org

2 Kelly KM, Shetty MK, Fregnani JHTG. Breast cancer screening and cervical cancer prevention in developing countries: strategies for the future. Breast Gynecol. Cancers XV, 301-329 (2013).

3 National Comprehensive Cancer Network. Breast Cancer Clinical Practice Guidelines in Oncology. J. Natl Compr. Canc. Netw. 1, 148-188 (2003). agents to breast cancer cells. Similarly, fibrinogengraft-poly ( $N$-vinyl caprolactam) loaded with these dual drugs were reported to have efficient delivery properties toward breast cancer cells in vitro $[15,16]$.

Fibrinogen-based nanosystems can be utilized not only for therapy but also for imaging purposes as well. A multifunctional fibrinogen nanoparticle was reported by Sanoj et al. for simultaneous therapy and imaging of breast cancer cells in vitro [17]. They have loaded a potent chemotherapeutic agent paclitaxel to fibrinogen-coated $\mathrm{CdTe} / \mathrm{ZnTe}$ quantum dots (QDs) that aid in cancer cell imaging. Fibrinogen coating has significantly reduced the toxicity induced by the bare QDs which make it more cytocompatible and also provide $\alpha 5 \beta 1$ Integrin receptor targeting to breast cancer cells. $\alpha 5 \beta 1$ positive cancer cells like MCF-7 and Hela cells showed significant localization of these fibrinogen-coated QDs compared with that of $\alpha 5 \beta 1$-ve L929 and HT-29 cells. So the synthesized paclitaxel-fibrinogen-coated yellow QDs were reported to have a bifunctional, imaging and therapeutic effect on breast cancer cells [17].

\section{Future prospects}

The strategy of using thermo-responsive fibrinogen nanogels as a novel drug delivery strategy for breast cancer is yet to be exploited fully. The current in vitro research shows its potential in cancer therapeutics. Further in vivo studies using breast cancer models need to be done to check its efficacy in breast cancer.

\section{Financial \& competing interests disclosure}

The authors are grateful for the financial support from Department of Biotechnology (DBT), Government of India (funding reference number BT/PR10850/NNT/28/127/2008). Author S Maya is thankful to Council of Scientific and Industrial Research (CSIR), India for providing Senior Research Fellowship (SRF Award number 9/963 (0012) 2K11-EMR-I). The authors have no other relevant affiliations or financial involvement with any organization or entity with a financial interest in or financial conflict with the subject matter or materials discussed in the manuscript apart from those disclosed.

No writing assistance was utilized in the production of this manuscript.

4 Maya S, Sarmento B, Nair A, Rejinold NS, Nair SV, Jayakumar R. Smart stimuli sensitive nanogels in cancer drug delivery and imaging: a review. Curr. Pharm. Design. 19, 7203-7218 (2013).

5 Murali MY, Meena J, Subhash CC. Design and engineering of nanogels for cancer treatment. Drug Discov. Today 16, 457-463 (2011).

6 Jung KO, Ray D, Daniel JS, Krzysztof M. The development of microgels/nanogels for drug delivery applications. Prog. Polym. Sci. 33, 448-477 (2008). 
Thaski J, Opolski A, Wietrzyk J, Goarski A, Radzikowski C. Cytotoxic and antitumor effect of fibrinogen-methotrexate conjugate. Cancer Lett. 148, 189-195 (2000).

8 Jakate AS, Einhaus CM, DeAnglis AP, Retzinger GS, Desai PB. Preparation, characterization and preliminary application of fibrinogen-coated olive oil droplets for the targeted delivery of docetaxel to solid malignancies. Cancer Res. 73, 7314-7320 (2003).

9 Rejinold NS, Muthunarayanan M, Deepa N, Chennazhi KP, Nair SV, Jayakumar R. Development of novel fibrinogen nanoparticles by two-step co-acervation method. Int. J. Biol. Macromol. 47, 37-43 (2010).

10 Rejinold NS, Muthunarayanan M, Chennazhi KP, Nair SV, R. Jayakumar R. Curcumin loaded fibrinogen nanoparticles for cancer drug delivery. J. Biomed. Nanotechnol. 7, 1-14 (2011).

11 Rejinold NS, Muthunarayanan M, Chennazhi KP, Nair SV, R. Jayakumar R. 5-Fluorouracil loaded fibrinogen nanoparticles for cancer drug delivery applications. Int. J. Biol. Macromol. 48, 98-105 (2011).
12 Hallahan DE, Geng L, Cmelak AJ et al. Targeting drug delivery to radiation-induced neoantigens in tumor microvasculature. J. Control. Release 74, 183-191 (2001).

13 Hallahan DE, Geng L, Qu S et al. Integrin-mediated targeting of drug delivery to irradiated tumor blood vessels. Cancer Cell 3, 63-74 (2003).

14 Pelton R. Temperature-sensitive aqueous microgels. Adv. Colloid Interface Sci. 85, 1-33 (2000).

15 Rejinold NS, Baby T, Chennazhi KP, Jayakumar R. Dual drug encapsulated thermo-sensitive fibrinogen-graftpoly( $N$-isopropyl acrylamide) nanogels for breast cancer therapy. Coll. Surf. B. Biointerfaces 114, 209-217 (2014).

16 Rejinold NS, Baby T, Chennazhi KP, Jayakumar R. Multi drug loaded thermo-responsive fibrinogen-graft-poly $(N$ vinyl caprolactam) nanogels for breast cancer drug delivery. J. Biomed. Nanotechnol. 11, 392-492 (2015).

17 Rejinold NS, Baby T, Nair SV, Jayakumar R. Paclitaxel loaded fibrinogen coated $\mathrm{CdTe} / \mathrm{ZnTe}$ core shell nanoparticles for targeted imaging and drug delivery to breast cancer cells. J. Biomed. Nanotechnol. 9, 1-15 (2013). 